\title{
Komplementärmedizin im politischen Prozess
}

\section{Schweizer Bevölkerung stimmt über Verfassungsartikel «Zukunft mit Komplementärmedizin" ab}

\author{
Lukas Rist ${ }^{a, b}$ Herbert Schwabl ${ }^{c, d}$ \\ a Paracelsus-Spital, Richterswil, \\ ' Geschäftsstelle Verfassungsartikel "Zukunft mit Komplementärmedizin», \\ ${ }^{\circ} \mathrm{ffg}$ - forum ganzheitsmedizin, Eschenz, \\ dPadma AG, Schwerzenbach, Schweiz
}

Die Nachfrage nach komplementärmedizinischen Behandlungen ist in der Schweiz groß. Gemäß einer repräsentativen Umfrage machen 57\% der Bevölkerung davon Gebrauch [1, 2]. Rund $40 \%$ der ärztlichen Grundversorger der Schweiz sind ganz oder teilweise komplementärmedizinisch tätig. Nach einer unveröffentlichten Studie der Kollegialen Instanz für Komplementärmedizin der Universität Bern ziehen weitere $40 \%$ der Allgemeinmediziner komplementärmedizinische Verfahren bei Bedarf hinzu [3]. Fast jeder vierte frei praktizierende Arzt ist über eine Fachgesellschaft Mitglied der UNION der komplementärmedizinischen Ärzteorganisationen [4]. Dennoch wird die Komplementärmedizin offiziell bis heute nicht als integraler Bestandteil des Schweizer Gesundheitswesens anerkannt.

Vorgeschichte - eine wissenschaftliche Odyssee

Ab 1. Juli 1999 wurden fünf komplementärmedizinische Methoden (anthroposophische Medizin, Homöopathie, Neuraltherapie, Phytotherapie und traditionelle chinesische Medizin) befristet für 6 Jahre in die Krankengrundversicherung aufgenommen und Leistungen von Ärzten mit entsprechendem Fähigkeitsausweis vergütet. Die Entscheidung über den weiteren Verbleib dieser fünf Verfahren in der Grundversicherung war an den wissenschaftlichen Nachweis ihrer Wirksamkeit, Zweckmäßigkeit und Wirtschaftlichkeit (WZW) gebunden, die vom «Programm Evaluation Komplementärmedizin» (PEK) geprüft werden sollten.

Das PEK wurde 2006 abgeschlossen. Sowohl der interne Schlussbericht als auch die teilweise veröffentlichten Forschungsberichte zeigten, dass die fünf Methoden die WZWKriterien erfüllen [5-7]. Der Schlussbericht ging danach von den Wissenschaftlern zur Verwaltungsbehörde, zum Bundesamt für Gesundheit.

Im von der Verwaltung erstellten Schlussbericht [8] wurden die positiven Empfehlungen des PEK-Bewertungsausschusses ersatzlos gestrichen $[9,10]$. Beibehalten wurden die grundsätzlich positiven Bewertungen aller fünf Methoden [4]. Noch vor Abschluss des PEK hatte der zuständige Gesundheitsminister die fünf Verfahren gemäß Entscheid vom 3. Juni 2005 wieder aus Leistungskatalog der Krankengrundversicherung ausgeschlossen $[11,12]$. Folgerichtig hält die parlamentarische Geschäftsprüfungskommission in ihrem Jahresbericht vom 23. Januar 2009 fest, dass bei dem Vorgehen des Gesundheitsministers eher politische als objektive Erwägungen entscheidend waren [13].

Eidgenössische Volksinitiative «Ja zur Komplementärmedizin» In den letzten Jahren wurde deutlich, dass in der Schweiz weder Bund noch Kantone die Komplementärmedizin fördern wollen, bestehende Gesetzesartikel wurden sehr restriktiv ausgelegt. Dies hat den Dachverband «ffg - Forum für Ganzheitsmedizin» im Jahr 2004 dazu bewogen, mit dem Mittel der Volksinitiative einen neuen Artikel in die Schweizer Bundesverfassung zu bringen, der das Bestehen der Komplementärmedizin sichert:

Bundesverfassung Art. 118a (neu): Komplementärmedizin Bund und Kantone sorgen im Rahmen ihrer Zuständigkeiten für die umfassende Berücksichtigung der Komplementärmedizin.

Im Unterstützungskomitee der Initiative schlossen sich fast alle Schweizer komplementärmedizinischen Berufs- und Interessensgruppen zusammen: Ärzte, Heilpraktiker und Therapeuten, Apotheker, Drogisten, Pflegefachleute, Heilmittelhersteller und Patientenorganisationen. Um den sprachlichen Gepflogenheiten und dem Allgemeinverständnis der Schweizer Bevölkerung entgegenzukommen, einigte man sich auf den einheitlichen Begriff «Komplementärmedizin» (französisch: médecines complémentaires, italienisch: medicina complementare), der die etablierten Varianten und Definitionen der komplementären und alternativen Medizin mit einschließt. Aktuelle Informationen finden sich auf der Website www.ja-zur-komplementaermedizin.ch.

\section{KARGER}

Fax +497614520714

Information@Karger.de

www.karger.com (c) 2009 S. Karger GmbH, Freiburg

Accessible online at:

www.karger.com/fok 
Der politische Prozess - Exkurs in die direkte Demokratie der Schweiz

Volksinitiativen sind eine Besonderheit der Schweizer direkten Demokratie, durch die die Wahlberechtigten eine Volksabstimmung über Änderungen der Schweizerischen Bundesverfassung verlangen können. Die Volksinitiative «Ja zur Komplementärmedizin» wurde am 21. September 2004 mit einer Unterschriftensammlung lanciert, bei der innerhalb von 12 Monaten knapp 140000 Unterschriften geleistet wurden. Am 4. Oktober 2005 hat die Bundeskanzlei offiziell das Verfahren für die Volksabstimmung eingeleitet [14]. Regierung (Bundesrat) und Parlament konnten nun den Verfassungstext beraten und den Wählern zur Annahme oder zur Ablehnung empfehlen. Der Bundesrat hat sich im August 2006 in seiner Botschaft gegen den vorgeschlagenen Artikel ausgesprochen [15]. Auch dem Parlament ging der Verfassungstext mit der Wortwahl der «umfassenden Berücksichtigung der Komplementärmedizin» wohl zu weit; der Nationalrat hat die Vorlage im September 2007 zur Ablehnung empfohlen [16]. Das Parlament kann jedoch einer Volksinitiative einen so genannten «direkten Gegenvorschlag» gegenüberstellen, wozu es einen eigenen Verfassungsartikel vorschlägt. Dies wurde in der kleinen Parlamentskammer (Ständerat, Kammer der Kantonsvertreter) realisiert: auf Initiative von Ständerat Rolf Büttiker kam der direkte Gegenvorschlag «Zukunft mit Komplementärmedizin» zustande:

Bundesverfassung Art. 118a (neu): Komplementärmedizin

Bund und Kantone sorgen im Rahmen ihrer Zuständigkeiten für die Berücksichtigung der Komplementärmedizin.

Vom ursprünglichen Text unterscheidet sich der Gegenvorschlag lediglich dadurch, dass das Wort «umfassend» fehlt. Damit konnten die Bedenken vieler Parlamentarier vor unabsehbaren Kostenfolgen aus dem Weg geräumt werden. In der Schlussabstimmung am 3. Oktober 2008 wurde der Gegenvorschlag von 78,5\% aller National- und Ständeräte genehmigt und dem Schweizer Stimmvolk zur Annahme empfohlen [17]. Das Initiativkomitee hat daraufhin beschlossen, die ursprüngliche Volksinitiative zurückzuziehen und den parlamentarischen Gegenvorschlag zu unterstützen. So konnte vermieden werden, dass die Wähler über zwei praktisch gleichlautende Vorlagen entscheiden müssen, was die Gefahr in sich birgt, dass die Stimmberechtigten verunsichert werden und eine ablehnende Haltung einnehmen.

Es gilt zu bedenken, dass als große Hürde für jede Verfassungsänderung neben der absoluten Mehrheit auch die zustimmende Mehrheit der Kantone erforderlich ist. Historisch gesehen haben parlamentarische Gegenvorschläge grundsätzlich bessere Chancen von Volk und Kantonen angenommen zu werden: seit 1891 kamen nur 9\% aller Volksinitiativen durch, verglichen mit $37 \%$ der direkten Gegenvorschläge.

\section{Die politischen Kernforderungen}

Der parlamentarische Gegenvorschlag kommt unter dem Namen «Zukunft mit Komplementärmedizin» am 17. Mai 2009 zur Volksabstimmung [21]. Der Verfassungsartikel geht vom Verständnis mündiger, aufgeklärter Bürgerinnen und Bürger aus und soll für sie als eigenverantwortliche Patienten oder Angehörige die freie Therapiewahl in der Krankenversorgung, Rehabilitation und Gesundheitsvorsorge unter Einbeziehung komplementärer Methoden gewährleisten. Der Umgang mit Gesundheit und Krankheit wird als Teilnahme an einem das Leben gestaltenden, aktiven Prozess verstanden - eine Einstellung die z.B. bei vielen chronisch Kranken zu beobachten ist. Um diese aktive Rolle wahrzunehmen, müssen die Bürger umfassend über die begründeten Therapieverfahren informiert sein. Freie Therapiewahl setzt voraus, dass der Komplementärmedizin ausdrücklich und aufgrund von definierten Rahmenbedingungen der Platz eingeräumt wird, der ihr in einer pluralistischen Gesellschaft zusteht. Ergänzend dazu steht das Bild von qualifizierten Fachpersonen, die ihre Patienten in den Heilungsprozess mit einbeziehen. Verantwortungsbewusstes Handeln auf beiden Seiten ist das Stichwort. Im Folgenden werden die für die professionellen Fachgruppen relevanten politischen Forderungen aufgeführt:

\section{Fünf ärztliche Richtungen wieder in die Grundversicherung} Ärztliche Leistungen in anthroposophischer Medizin, Homöopathie, Neuraltherapie, Phytotherapie und traditioneller chinesischer Medizin müssen wieder in die Grundversicherung aufgenommen werden. Dies bedingt keine gesetzlichen Änderungen, sondern ist der korrekte Vollzug des Krankenversicherungsgesetzes (KVG). Voraussetzung für die Vergütung durch die Grundversicherung ist ein anerkannter Fähigkeitsausweis für die jeweilige Disziplin. Von administrativer Seite braucht es dazu weder neue Vorschriften noch zusätzliche Tarifpositionen. Eine Ausweitung des Leistungskatalogs durch die Aufnahme weiterer Therapierichtungen ist dabei nicht vorgesehen, ebenso werden nichtärztliche Therapeuten weiterhin nur über die Zusatzversicherungen abrechnen; diesbezüglich sind also keine Zusatzkosten zu erwarten.

\section{Nationale Diplome für nichtärztliche Therapeuten}

Für viele Patienten sind nichtärztliche Therapeuten zu einer ersten Anlaufstelle bei Krankheiten geworden. Um die Patientensicherheit zu gewährleisten, ist es deshalb erforderlich, dass es künftig für nichtärztliche Therapeuten Diplome und Berufsausübungsbewilligungen gibt. Heute kann in sechs Kantonen jede Person ohne Ausbildung oder Berufserfahrung eine Praxis eröffnen. Von den Therapeutenverbänden selbst wird dieser Zustand kritisiert. Sie fordern im Interesse der Mitglieder, aber auch der Patienten und der Öffentlichkeit einheitliche Diplome, wie sie das Berufsbildungsgesetz vorsieht. Die Kosten für Ausbildungen, Prüfungen und Diplome werden die Therapeuten selber tragen.

\section{Integration in Lehre und Forschung}

Die Komplementärmedizin ist in Lehre und Forschung vermehrt zu berücksichtigen. Jeder Arzt und jede Ärztin soll in der Ausbildung wenigstens die Grundsätze von Heilmetho- 
den kennenlernen, die von einer Mehrheit der Bevölkerung nachgefragt werden. Die Verordnung über die Prüfungen für Ärzte verlangt, dass sich die Ärzteausbildung an den Bedürfnissen der Bevölkerung orientiert [25]. Daraus leitet sich ab, dass zumindest für die wichtigsten Methoden ein universitäres Angebot einzurichten ist. Dieses ist zu ergänzen durch komplementärmedizinische Angebote in der Pharmazie, insbesondere in der Pflanzenheilkunde. Durch den entsprechenden verfassungsmäßigen Auftrag ist der Rahmen für eine verstärkte Berücksichtigung komplementärer medizinischer und pharmazeutischer Methoden bei Lehre und Forschung an den verschiedenen Schweizer Universitätsstandorten geschaffen. Ferner soll sich die Schweizer Delegation als assoziiertes Mitglied bei den Forschungsförderungsprogrammen der Europäischen Union für die Berücksichtigung der Komplementärmedizin einsetzen.

\section{Wahrung des bewährten Heilmittelschatzes}

Das Schweizer Heilmittelgesetz sieht im Artikel 14 eine vereinfachte Zulassung für Arzneimittel der Komplementärmedizin vor [15]. Entgegen dieser Bestimmung hat die Schweizer Zulassungsbehörde Swissmedic den bürokratischen Formalismus gestärkt und verlangt zum Teil hohe Gebühren für die Einreichung und Bearbeitung von Gesuchen. In der Folge verschwinden in der Schweiz immer mehr komplementärmedizinische Heilmittel vom Markt. Notwendig ist neben den formellen und finanziellen Erleichterungen eine Regelung für traditionelle sowie für die auslaufenden kantonal zugelassenen Arzneimittel.

\section{Ausblick - Volksabstimmung «Zukunft mit Komplementärmedizin»}

Das Team, welches die Kampagne zur Abstimmung koordiniert, ist nun gefordert, alle möglichen Kräfte für den 17. Mai 2009 zu mobilisieren. Der finanzielle und personelle Aufwand zur Durchführung einer professionellen Kampagne ist enorm. Auch wenn auf die Mithilfe und Unterstützung sämtlicher an der Komplementärmedizin interessierten Verbände und Fachleute gezählt werden kann, ist die Abstimmung noch nicht gewonnen. Insbesondere gilt es, die Befürworter zu motivieren, auch wirklich an der Abstimmung teilzunehmen.

Soweit man den politischen Prozess bis anhin als Erfolg ansehen kann, zeigt er aber auch, dass nur das gemeinsame und geschlossene Auftreten aller Interessenvertreter diesen politischen Druck aufbauen konnte. Diese Strategie könnte auch ein beispielgebender Erfolgsfaktor für ähnliche Vorhaben in anderen Ländern sein.

Die Chancen für die Annahme der Vorlage «Zukunft mit Komplementärmedizin» stehen gut. Mehrere repräsentative Umfragen zeigen, dass der Ja-Stimmenanteil hoch sein dürfte $[26,27]$. Doch der Widerstand der politischen Gegner wird sich zweifellos noch formieren und darf nicht unterschätzt werden. Mit dem Verfassungsartikel wäre grundsätzlich geklärt, dass die Komplementärmedizin nicht nur geduldet wird, sondern integraler Bestandteil des Schweizer Gesundheitswesens ist. Damit wäre die Schweiz das erste Land, in dem die Komplementärmedizin in der Verfassung verankert ist und hätte somit eine Vorreiterrolle in Europa.

\section{Literatur}

$>_{1}$ Wolf U, Maxion-Bergemann S, Bornhöft G, Matthiessen PF: Use of complementary medicine in Switzerland. Forsch Komplementmed 2006;13(suppl 2):4-6.

2 DemoScope Bericht: Umfrage Komplementärmedizin. Bern, Demoscope AG, 2007.

3 Fritschi J: Soll sich die FMH für die Erhaltung oder sogar für die Förderung komplementärmedizinischer Angebote bei ihren Mitgliedern einsetzen? Schweiz Ärzteztg 2005;86:27-30.

4 Fritschi J: Zukunft mit Komplementärmedizin Schweiz Ärzteztg 2009;90:16-18.

$\checkmark 5$ Kienle GS, Kiene H, Albonico HU: Anthroposophische Medizin: Health Technology Assessment Bericht - Kurzfassung. Forsch Komplementmed 2006;13(suppl 2):7-18.

6 Bornhöft G, Wolf U, von Ammon K, Righetti M, Maxion-Bergemann S, Baumgartner S, Thurneysen A, Matthiessen PF: Wirksamkeit, Sicherheit und Wirtschaftlichkeit von Homöopathie unter Alltagsbedingungen - Zusammenfassung des Health Technology Assessment Berichts. Forsch Komplementmed 2006;13(suppl 2):19-29.

7 Maxion-Bergemann S, Bornhöft G, Sonderegger E, Renfer A, Matthiessen PF, Wolf U: Traditionelle Chinesische Medizin (Phytotherapie): Health Technology Assessment Bericht - ausgewählte Aspekte. Forsch Komplementmed 2006;13(suppl 2):30-41.
8 Schlussbericht Programm Evaluation Komplementärmedizin (PEK) vom 24.04.2006. www.bag.admin. ch/themen/krankenversicherung/00263/00264/04102/ index.html.

9 Walach H, Linde K, Eichenberger R, Stalder H, Børlum Kristensen F, Kleijnen J; Swiss Complementary Medicine Evaluation Programme: Summary consensus statement of the Review Board of the Swiss Complementary Medicine Evaluation Programme (Programm Evaluation Komplementärmedizin, PEK) regarding the PEK process and products. Homeopathy 2006;95:28-30.

10 Heusser P: PEK und BAG: Probleme beim Programm Evaluation Komplementärmedizin. Schweiz Ärzteztg 2006;87:899-903.

11 Walach H: The Swiss program for the evaluation of complementary medicine (PEK). J Altern Complement Med 2006;12:231-232.

12 Schweizerische Eidgenossenschaft, Bundesamt für Gesundheit: Medienmitteilung. Bern, 03.06.2005. www.bag.admin.ch/aktuell/00718/01220/index. html lang $=$ de\&msg-id $=3740$.

13 Schweizer Parlament, Geschäftsprüfungskommissionen und der Geschäftsprüfungsdelegation der eidgenössischen Räte: Jahresbericht 2008. www.parlament. ch/D/Documents/gpk-n-s-jahresbericht-2008-d.pdf.

14 Schweizerische Eidgenossenschaft, Bundeskanzlei: Bekanntmachung der Departemente und der Ämter. Bern, 04.10.2005. Eidgenössische Volksinitiative «Ja zur Komplementärmedizin» Zustandekommen. www.admin.ch/ch/d/ff/2005/6001.pdf.
15 Schweizerischer Bundesrat: Botschaft über die Volksinitiative «Ja zur Komplementärmedizin». Bern, 30.08.2006. www.admin.ch/ch/d/ff/2006/7591. pdf.

16 Schweizer Nationalrat: Bundesbeschluss über die Volksinitiative «Ja zur Komplementärmedizin». Herbstsession 2007, Dritte Sitzung, Amtliches Bulletin. Bern, 19.09.07. www.parlament.ch/ab/frameset/d/n/4718/252001/d_n_4718_252001_252002.htm

17 Schweizer Parlament: Bundesbeschluss über die Volksinitiative «Ja zur Komplementärmedizin». Bern, 03.10.2008. www.admin.ch/ch/d/ff/2008/8229. pdf.

18 Schweizerische Eidgenossenschaft: Bundeskanzlei Vorlagen zur Volksabstimmung vom 17. Mai 2009. Bern, 14.01.2009. www.bk.admin.ch/aktuell/media/ 03238/index.html?lang = de\&msg-id $=24831$.

19 Schweizerischer Bundesrat: Verordnung vom 19. November 1980 über die Prüfungen für Ärzte (SR 811.112.2): www.admin.ch/ch/d/sr/c811_112_2.html. 20 Schweizer Heilmittelgesetz (Bundesgesetz über Arzneimittel und Medizinprodukte): www.admin. $\mathrm{ch} / \mathrm{ch} / \mathrm{d} / \mathrm{sr} / \mathrm{c} 812 \_21 . h t m l$.

21 Santésuisse: Bericht zur sondage santé. Bern, 2008. www.santesuisse.ch/datasheets/files/200809041104034. pdf.

22 Interpharma Info-Center: gfs Gesundheitsmonitor. Bern, 2008. www.interpharma.ch/de/4768.asp. 